\title{
Theory of spin-polarized transport in ferromagnet-semiconductor structures: Unified description of ballistic and diffusive transport
}

\author{
R. Lipperheide, U. Wille ${ }^{1}$ \\ Abteilung Theoretische Physik, Hahn-Meitner-Institut Berlin, \\ Glienicker Str. 100, D-14109 Berlin, Germany
}

\begin{abstract}
A theory of spin-polarized electron transport in ferromagnet-semiconductor heterostructures, based on a unified semiclassical description of ballistic and diffusive transport in semiconductors, is outlined. The aim is to provide a framework for studying the interplay of spin relaxation and transport mechanism in spintronic devices. Transport inside the (nondegenerate) semiconductor is described in terms of a thermoballistic current, in which electrons move ballistically in the electric field arising from internal and external electrostatic potentials, and are thermalized at randomly distributed equilibration points. Spin relaxation is allowed to take place during the ballistic motion. For arbitrary potential profile and arbitrary values of the momentum and spin relaxation lengths, an integral equation for a spin transport function determining the spin polarization in the semiconductor is derived. For field-driven transport in a homogeneous semiconductor, the integral equation can be converted into a second-order differential equation that generalizes the spin drift-diffusion equation. The spin polarization in ferromagnet-semiconductor structures is obtained by matching the spin-resolved chemical potentials at the interfaces, with allowance for spin-selective interface resistances. Illustrative examples are considered.
\end{abstract}

PACS: 72.25.-b, 72.25.Hg, 73.40.Cg, 73.40.Sx

Keywords: Theory; Heterostructures; Spin-polarized transport; Transport mechanisms; Spintronics

\footnotetext{
${ }^{1}$ Corresponding author. Phone: ++49-30-80622685; FAX: ++49-30-80622098; E-mail: wille@hmi.de.
} 


\section{Introduction}

In spintronics research, particular emphasis is currently placed on the study of spinpolarized electron transport in heterostructures formed of a nonmagnetic semiconductor and two (metallic or semiconducting) ferromagnetic contacts $[1,2,3,4]$. For the actual design of spintronic devices, a detailed theoretical understanding of this kind of transport problem is indispensable. Several pertinent studies have been performed so far, which mostly rely on the drift-diffusion model. A number of important results have emerged. (i) For an interface between a metallic ferromagnet and a semiconductor without spinselective interface resistance, the injected current spin polarization is predicted to be very low owing to the large conductivity mismatch [5]. (ii) Spin-selective interface resistances arising from tunnel barriers or Schottky barriers can greatly enhance the injection efficiency $[6,7,8,9,10,11]$. (iii) A similar effect is to be expected when a sufficiently high electric field is applied across the semiconductor [12]. (iv) Under conditions where, in the semiconductor, ballistic transport prevails over drift-diffusion, the injection efficiency is controlled by the Sharvin interface resistance [13] unless spin-selective interface resistances are introduced.

While the theory of spin-polarized electron transport in ferromagnet-semiconductor heterostructures has reached a level of considerable sophistication, it appears that certain aspects of the semiconductor part of the transport problem require a more systematic, unified treatment, such as the interplay of spin relaxation and transport mechanism all the way from the diffusive to the ballistic regime, and the effect of the detailed shape of the electrostatic potential profile. In this paper, we outline the principal ideas of a theory that meets these requirements. For illustrative purposes, we present calculated results for the position dependence of the zero-bias current spin polarization along a heterostructure as well as for the injected polarization as a function of bias. A detailed account of the formal development as well as specific applications of our theory will be published elsewhere [14].

\section{Thermoballistic current}

Our treatment of spin-polarized electron transport relies on our previously formulated unified semiclassical description of (spinless) electron transport in parallel-plane semiconductor structures [15]. The description is based on the concept of a "thermoballistic electron current" which combines elements of the ballistic and diffusive transport mechanisms. 
Here, we briefly summarize this concept.

Assuming a one-dimensional geometry, we consider a (nondegenerate) semiconducting sample enclosed between two plane-parallel ferromagnetic contacts at $x=x_{1}$ and $x=x_{2}$, respectively, so that $S=x_{2}-x_{1}$ is the sample length (see Fig. 1). The electron current density $J\left(x^{\prime}, x^{\prime \prime}\right)$ across the "ballistic interval" $\left[x^{\prime}, x^{\prime \prime}\right]$ between two equilibration points $x^{\prime}$ and $x^{\prime \prime}$ is given by

$$
J\left(x^{\prime}, x^{\prime \prime}\right)=v_{e} N_{c} e^{-\beta E_{c}^{m}\left(x^{\prime}, x^{\prime \prime}\right)}\left[e^{\beta \mu\left(x^{\prime}\right)}-e^{\beta \mu\left(x^{\prime \prime}\right)}\right]
$$

$\left(x_{1} \leq x^{\prime}<x^{\prime \prime} \leq x_{2}\right)$, which is the difference of the ballistic current injected into the interval at its left end at $x^{\prime}$ and the analogous current injected at its right end at $x^{\prime \prime}$. The function $\mu(x)$ is the chemical potential at the equilibration point $x$. Furthermore, $v_{e}=\left(2 \pi m^{*} \beta\right)^{-1 / 2}$ is the emission velocity, $N_{c}=2\left(2 \pi m^{*} / \beta h^{2}\right)^{3 / 2}$ is the effective density of states at the conduction band edge, $m^{*}$ is the effective mass of the electrons, and $\beta=\left(k_{B} T\right)^{-1}$. The current (1) contains only the transmitted electrons, i.e., those with sufficient energy to surmount the potential barrier

$$
\hat{E}_{c}^{m}\left(x^{\prime}, x^{\prime \prime}\right)=E_{c}^{m}\left(x^{\prime}, x^{\prime \prime}\right)-E_{c}^{0},
$$

where $E_{c}^{m}\left(x^{\prime}, x^{\prime \prime}\right)$ is the maximum value of the potential profile $E_{c}(x)$ in the interval $\left[x^{\prime}, x^{\prime \prime}\right]$, and $E_{c}^{0}$ is its overall minimum across the sample. The profile $E_{c}(x)$ comprises the (equilibrium) conduction band edge potential and the external electrostatic potential.

From the ballistic electron current $J\left(x^{\prime}, x^{\prime \prime}\right)$, we construct the thermoballistic current $J(x)$ at position $x$ inside the sample by summing up the contributions from all intervals $\left[x^{\prime}, x^{\prime \prime}\right]$ for which $x_{1} \leq x^{\prime}<x<x^{\prime \prime} \leq x_{2}$, each weighted with the probability of occurrence of the interval. For simplicity, we here take this probability in its one-dimensional form $\exp \left(-\left|x^{\prime \prime}-x^{\prime}\right| / l\right)$, where $l$ is the mean free path for momentum relaxation (momentum relaxation length). We then have

$$
\begin{aligned}
& J(x)=v_{e} N_{c} e^{-\beta E_{c}^{0}}\left\{w\left(x_{1}, x_{2} ; l\right)\left[e^{\beta \mu_{1}}-e^{\beta \mu_{2}}\right]+\int_{x_{1}}^{x} \frac{d x^{\prime}}{l} w\left(x^{\prime}, x_{2} ; l\right)\left[e^{\beta \mu\left(x^{\prime}\right)}-e^{\beta \mu_{2}}\right]\right. \\
& \left.+\int_{x}^{x_{2}} \frac{d x^{\prime}}{l} w\left(x_{1}, x^{\prime} ; l\right)\left[e^{\beta \mu_{1}}-e^{\beta \mu\left(x^{\prime}\right)}\right]+\int_{x_{1}}^{x} \frac{d x^{\prime}}{l} \int_{x}^{x_{2}} \frac{d x^{\prime \prime}}{l} w\left(x^{\prime}, x^{\prime \prime} ; l\right)\left[e^{\beta \mu\left(x^{\prime}\right)}-e^{\beta \mu\left(x^{\prime \prime}\right)}\right]\right\},
\end{aligned}
$$

where

$$
w\left(x^{\prime}, x^{\prime \prime} ; l\right)=e^{-\left|x^{\prime \prime}-x^{\prime}\right| / l} e^{-\beta \hat{E}_{c}^{m}\left(x^{\prime}, x^{\prime \prime}\right)} .
$$


The quantities $\mu_{1,2}=\mu\left(x_{1,2}\right)$ are the chemical potentials on the contact side of the interfaces, i.e., immediately outside of the sample. The thermoballistic current $J(x)$ is not conserved by itself; however, when averaged over the sample, it yields $[14,15]$ the physical electron current $J$,

$$
\frac{1}{x_{2}-x_{1}} \int_{x_{1}}^{x_{2}} d x J(x)=J
$$

Furthermore, the thermoballistic current entering at one end of the sample must equal the current leaving at the other end [14],

$$
J\left(x_{1}^{+}\right)=J\left(x_{2}^{-}\right)
$$

Substituting expression (3) in condition (5), we can derive Volterra-type integral equations for two auxiliary functions $\chi_{1}(x)$ and $\chi_{2}(x)$, which are distinguished by discontinuities at $x=x_{1}$ and $x=x_{2}$, respectively. Using condition (6), we determine from $\chi_{1}(x)$ and $\chi_{2}(x)$ a unique chemical potential $\mu(x)$. It exhibits discontinuities at both interfaces, which are related to the Sharvin interface resistance [16]. From $\mu(x)$, the thermoballistic current $J(x)$ is calculated via Eq. (3). The current-voltage characteristic is obtained for arbitrary values of $l$ and arbitrary $E_{c}(x)$ in terms of a "reduced resistance" $\tilde{\chi}$ composed of $\chi_{1}\left(x_{2}\right)$ and $\chi_{2}\left(x_{1}\right)$. The thermoballistic density $n(x)$ of electrons making up the current $J(x)$ is constructed in a similar way.

For electron transport in a homogeneous semiconductor driven by a (constant) electric field of strength $\mathcal{E}$, the thermoballistic current $J(x)$ and density $n(x)$ can be expressed essentially in terms of the dimensionless parameters $x / S, l / S$, and $\epsilon S$, where $\epsilon=\beta e|\mathcal{E}|$. In Fig. 2, we show the dependence of $n(x)$ on $x / S$ for $\epsilon S=1$ and various values of $l / S$. The initial decrease of $n(x)$ with increasing $x / S$ is a ballistic effect that reflects the increase of the electron velocity in the electric field and becomes more pronounced as $l / S$ becomes larger. The corresponding thermoballistic current $J(x)$ turns out to differ only insignificantly from the physical current $J$.

\section{Spin-polarized transport in semiconductors}

We now extend the unified description by allowing spin relaxation to take place during the electron motion across the ballistic intervals. The off-equilibrium spin-polarized current $J_{-}(x)=J_{\uparrow}(x)-J_{\downarrow}(x)$ is connected with the off-equilibrium spin-polarized density $n_{-}(x)=$ 
$n_{\uparrow}(x)-n_{\downarrow}(x)$ through the balance equation

$$
\frac{d J_{-}(x)}{d x}+\frac{n_{-}(x)}{\tau_{s}}=0
$$

where $\tau_{s}$ is the spin relaxation time. Using this equation in the ballistic transport regime, in which $J_{-}(x)=2 v_{e} n_{-}(x)$, we construct the off-equilibrium ballistic spin-polarized current $J_{-}\left(x^{\prime}, x^{\prime \prime} ; x\right)$ at position $x$ in the ballistic interval $\left[x^{\prime}, x^{\prime \prime}\right]$, obtaining

$$
J_{-}\left(x^{\prime}, x^{\prime \prime} ; x\right)=v_{e} N_{c} e^{-\beta \hat{E}_{c}^{m}\left(x^{\prime}, x^{\prime \prime}\right)}\left[A\left(x^{\prime}\right) e^{-\left(x-x^{\prime}\right) / l_{s}}-A\left(x^{\prime \prime}\right) e^{-\left(x^{\prime \prime}-x\right) / l_{s}}\right]
$$

$\left(x_{1} \leq x^{\prime}<x<x^{\prime \prime} \leq x_{2}\right)$, with the ballistic spin relaxation length $l_{s}=2 v_{e} \tau_{s}$. The parameter $l_{s}$ comprises the overall effect of the underlying microscopic spin relaxation mechanisms. The function $A(x)$ is the "spin transport function" defined as

$$
A\left(x^{\prime}\right)=e^{-\beta\left[E_{c}^{0}-\mu\left(x^{\prime}\right)\right]} \alpha_{-}\left(x^{\prime}\right)
$$

here, $\alpha_{-}\left(x^{\prime}\right)=\alpha_{\uparrow}\left(x^{\prime}\right)-\alpha_{\downarrow}\left(x^{\prime}\right)$ is the off-equilibrium "spin fraction excess" at the equilibration point $x^{\prime}$, which is defined in terms of the spin fractions $\alpha_{\uparrow \downarrow}\left(x^{\prime}\right)$, with $\alpha_{\uparrow}\left(x^{\prime}\right)+\alpha_{\downarrow}\left(x^{\prime}\right)=1$. The spin fractions are related to the spin-resolved thermoballistic chemical potentials $\mu_{\uparrow \downarrow}\left(x^{\prime}\right)$ via

$$
e^{\beta \mu_{\uparrow \downarrow}\left(x^{\prime}\right)}=e^{\beta \mu\left(x^{\prime}\right)} \alpha_{\uparrow \downarrow}\left(x^{\prime}\right)
$$

Proceeding as in the spinless case, we now sum the (weighted) contributions of the ballistic spin-polarized current (8) over all randomly distributed intervals $\left[x^{\prime}, x^{\prime \prime}\right]$. The resulting expression for the off-equilibrium thermoballistic spin-polarized current $J_{-}(x)$ is of the form (3), but with the terms in brackets therein replaced with those obtained by evaluating the bracketed term in Eq. (8) for the different cases. A similar expression is found for the off-equilibrium thermoballistic spin-polarized density $n_{-}(x)$.

Substituting the thermoballistic expressions for $J_{-}(x)$ and $n_{-}(x)$ in Eq. (7), we arrive at a linear, Fredholm-type integral equation for the spin transport function $A(x)$,

$$
\begin{aligned}
W\left(x_{1}, x ; l, l_{s}\right) A_{1} & +W\left(x, x_{2} ; l, l_{s}\right) A_{2} \\
& -W_{0}\left(x_{1}, x_{2} ; x ; l\right) A(x)+\int_{x_{1}}^{x_{2}} \frac{d x^{\prime}}{l} W\left(x^{\prime}, x ; l, l_{s}\right) A\left(x^{\prime}\right)=0
\end{aligned}
$$

where

$$
W\left(x^{\prime}, x^{\prime \prime} ; l, l_{s}\right)=w\left(x^{\prime}, x^{\prime \prime} ; l\right) e^{-\left|x^{\prime \prime}-x^{\prime}\right| / l_{s}},
$$




$$
W_{0}\left(x_{1}, x_{2} ; x ; l\right)=w\left(x_{1}, x ; l\right)+w\left(x, x_{2} ; l\right)+\int_{x_{1}}^{x_{2}} \frac{d x^{\prime}}{l} w\left(x^{\prime}, x ; l\right),
$$

and $A_{1,2}=A\left(x_{1,2}\right)$. The solution of the fundamental equation (11) for $x_{1}<x<x_{2}$ determines the spin-polarized electron transport inside the semiconducting sample, and is obtained in terms of the values of $A_{1}$ and $A_{2}$ on the contact side of the interfaces at the ends of the sample. The latter are given by the spin fraction excesses $\alpha_{1,2}=\alpha_{-}\left(x_{1,2}\right)$ and the chemical potentials $\mu_{1,2}=\mu\left(x_{1,2}\right)$ in the contacts via Eq. (9).

For field-driven transport in a homogeneous semiconductor, Eq. (11) can be converted, by twofold differentiation and elimination of the quantities $A_{1}$ and $A_{2}$, into a homogeneous integrodifferential equation. Within a judicious approximation, the latter equation can be reduced to a generalized spin drift-diffusion equation of the form

$$
b_{0}(x) \frac{d^{2} A(x)}{d x^{2}}+b_{1}(x) \frac{d A(x)}{d x}+b_{2}(x) A(x)=0,
$$

with coefficient functions $b_{i}(x)$ depending linearly on the function $\exp \left[-(\epsilon+1 / l)\left(x-x_{1}\right)\right]$. In the diffusive regime $l / l_{s} \ll 1$, where $b_{0}(x)=1, b_{1}(x)=\epsilon, b_{2}(x)=-1 /\left(l l_{s}\right)$, Eq. (14) reduces to the standard spin drift-diffusion equation [12] if there the intrinsic spin diffusion length $L$ is identified with $\sqrt{l l_{s}}$.

With $A(x)$ determined by solving the integral equation (11) or, in special cases, simplified equations like Eq. (14), we can evaluate the thermoballistic spin-polarized current $J_{-}(x)$ from the analogue of expression (3). Dividing by the (total) thermoballistic current $J(x)$ given by Eq. (3), we obtain the current spin polarization

$$
P_{J}(x)=\frac{J_{-}(x)}{J(x)} .
$$

The density spin polarization is calculated analogously.

\section{Ferromagnet-semiconductor heterostructures}

We now consider spin-polarized transport in heterostructures formed of a homogeneous, nonmagnetic semiconductor and two ferromagnetic contacts, which are treated as fully degenerate Fermi systems. Equating the splitting $\mu_{-}(x)=\mu_{\uparrow}(x)-\mu_{\downarrow}(x)$ of the spin-up and spin-down chemical potentials in the ferromagnets at $x=x_{1,2}$, respectively, with that of the semiconductor on the contact side of the interfaces, we find, using Eq. (10),

$$
\left[\mu_{-}\left(x_{1,2}\right)\right]_{\text {ferromagnet }}=\left[\mu_{-}\left(x_{1,2}\right)\right]_{\text {semiconductor }}=\frac{1}{\beta} \ln \left(\frac{1+\alpha_{1,2}}{1-\alpha_{1,2}}\right) \text {. }
$$


This condition allows the current spin polarizations $P_{J}\left(x_{1,2}\right)$ on the ferromagnet sides of the interfaces to be expressed through the spin fraction excesses $\alpha_{1,2}$. Neglecting spinflip scattering at the interfaces, we invoke continuity of the polarization at the interfaces. We then obtain two coupled nonlinear equations from which $\alpha_{1}$ and $\alpha_{2}$, and hence $J_{-}(x)$ and $P_{J}(x)$, can be calculated in terms of the material parameters characterizing the ferromagnets and the semiconductor, the bulk polarizations $P_{1}$ and $P_{2}$ in the left and right ferromagnet, respectively, and the physical current $J$. Spin-selective interface resistances can be introduced via discontinuities of the chemical-potential splitting $\mu_{-}(x)$ at the interfaces $[8,10,12]$.

In the case of zero bias, $\epsilon \rightarrow 0$, the solutions of Eq. (14) are $A(x) \propto \exp ( \pm x / L)$, and the position dependence of the current spin polarization inside the semiconductor is given by

$$
P_{J}(x)=\frac{2 v_{e} N_{c} \bar{l}}{L J}\left[C_{1} e^{-\left(x-x_{1}\right) / L}-C_{2} e^{-\left(x_{2}-x\right) / L}\right]
$$

where $L=\sqrt{\bar{l}_{s}}$ is the generalized spin diffusion length (or "polarization decay length"), and $\bar{l}=l l_{s} /\left(l+l_{s}\right)$. The coefficients $C_{1,2}$ are simple functions of the spin fraction excesses $\alpha_{1,2}$ which, in turn, are given explicitly in terms of the material parameters, the polarizations $P_{1,2}$, and the current $J$.

In Fig. 3, the zero-bias current spin polarization $P_{J}(x)$ for a symmetric ferromagnetsemiconductor-ferromagnet heterostructure with $S=1 \mu \mathrm{m}$ at $T=300 \mathrm{~K}$ is shown as a function of $x$ for various values of the momentum relaxation length $l$ and for zero as well as nonzero interface resistances. For the parameters of the ferromagnets, the values $10^{3} \Omega^{-1}$ $\mathrm{cm}^{-1}$ for the bulk conductivities, $60 \mathrm{~nm}$ for the spin diffusion lengths, and 0.5 for the bulk polarizations $P_{1,2}$ have been adopted from Ref. [12]. With a look at recent experiments on the spin dynamics in n-doped GaAs [17], the values $0.067 m_{e}$ for the effective electron mass $m^{*}, 2.0 \times 10^{18} \mathrm{~cm}^{-3}$ for the equilibrium electron density, and $1 \mu \mathrm{m}$ for the ballistic spin relaxation length $l_{s}$ have been chosen for the material parameters of the semiconductor. We are aware of the fact that, by considering a specific semiconducting material with fixed doping concentration, one essentially fixes the value of the momentum relaxation length $l$. Therefore, when varying $l$ in a fairly broad range, we assume the above parameter values (or, at least, their order of magnitude) to be representative for a class of semiconducting materials that differ in the strength of the impurity scattering and hence in the magnitude 
of $l$.

The momentum relaxation length $l$ affects the results shown in Fig. 3 in a twofold way. (i) It determines the conduction in the semiconductor and thus the conductivity mismatch with the ferromagnets. For small values of $l$, this mismatch is large, leading to a small injected current spin polarization $P_{J}(0)$. (ii) It determines the polarization decay length $L$, so that for small $l$ the polarization dies out rapidly inside the semiconductor. A substantial degree of polarization all along the semiconductor is achieved when the value of $l$ is increased up to a length of the order of the sample length, in which case the ballistic component becomes prevalent. Figure 3 also shows that, by introducing appropriately chosen spin-selective interface resistances, one may offset the suppression of the injected polarization due to the conductivity mismatch for small $l$; however, the rapid decay of the polarization inside the semiconductor cannot be prevented in this way.

For nonzero, constant electric field and $S / L \rightarrow \infty$, i.e., disregarding the effect of the right ferromagnet, we have for the current spin polarization $P_{J}\left(x_{1}\right)$ injected at the left interface

$$
P_{J}\left(x_{1}\right)=\tilde{\chi} \Gamma_{J} \alpha_{1}
$$

where $\tilde{\chi}$ is the reduced resistance entering the current-voltage characteristic, and the quantity $\Gamma_{J}$ involves the function $A(x)$, which is obtained by numerically solving Eq. (14). Since only the interface at $x=x_{1}$ enters into consideration, a single nonlinear equation has to be solved to determine the spin fraction excess $\alpha_{1}$ as a function of the polarization $P_{1}$.

In Fig. 4, we show the dependence of $P_{J}\left(x_{1}\right)$ on the electric-field parameter $\epsilon$ for various values of the momentum relaxation length $l$, the remaining parameter values being the same as in Fig. 3. In conformity with the drift-diffusion results of Ref. [12], the injected polarization generally rises with increasing $\epsilon$; however, as in Fig. 3, the main effect is due to the variation of $l$.

\section{Concluding remarks}

We have outlined the principal ideas of a theory of spin-polarized electron transport in ferromagnet-semiconductor heterostructures. It generalizes previous theoretical treatments based on the drift-diffusion model by introducing the momentum relaxation length in the semiconductor as a new degree of freedom, thus allowing a systematic study of the interplay 
of spin relaxation and transport mechanism. By considering illustrative examples, we have shown that the momentum relaxation length has a significant influence both on the polarization injected at a ferromagnet-semiconductor interface and on the decay of the polarization inside the semiconductor. To study in detail the influence of the transport mechanism on spin-polarized transport (in particular, when ballistic effects take over), the identification and design of classes of novel semiconducting materials is called for. In this way, new possibilities to improve the efficiency of spintronic devices may open up. 


\section{References}

[1] D. Grundler, Physics World 15, no. 4 (2002), p. 39.

[2] Semiconductor Spintronics and Quantum Computation, eds. D. D. Awschalom, D. Loss, and N. Samarth (Springer-Verlag, Berlin, 2002).

[3] G. Schmidt and L. W. Molenkamp, Semicond. Sci. Technol. 17 (2002) 310.

[4] I. Žutić, J. Fabian, and S. Das Sarma, Rev. Mod. Phys. 76 (2004) 323.

[5] G. Schmidt, D. Ferrand, L. W. Molenkamp, A. T. Filip, and B. J. van Wees, Phys. Rev. B 62 (2000) R4790.

[6] A. T. Filip, B. H. Hoving, F. J. Jedema, B. J. van Wees, B. Dutta, and S. Borghs, Phys. Rev. B 62 (2000) 9996.

[7] E. I. Rashba, Phys. Rev. B 62 (2000) R16267.

[8] D. L. Smith and R. N. Silver, Phys. Rev. B 64 (2001) 045323.

[9] A. Fert and H. Jaffrès, Phys. Rev. B 64 (2001) 184420.

[10] E. I. Rashba, Eur. Phys. J. B 29 (2002) 513.

[11] J. D. Albrecht and D. L. Smith, Phys. Rev. B 68 (2003) 035340.

[12] Z. G. Yu and M. E. Flatté, Phys. Rev. B 66 (2002) 235302.

[13] V. Ya. Kravchenko and E. I. Rashba, Phys. Rev. B 67 (2003) 121310(R).

[14] R. Lipperheide and U. Wille, to be published.

[15] R. Lipperheide and U. Wille, Phys. Rev. B 68 (2003) 115315.

[16] Yu. V. Sharvin, Zh. Eksp. Teor. Fiz. 48 (1965) 984 [Sov. Phys. JETP 21 (1965) 655].

[17] A. V. Kimel, F. Bentivegna, V. N. Gridnev, V. V. Pavlov, R. V. Pisarev, and Th. Rasing, Phys. Rev. B 63 (2001) 235201. 


\section{Figure Captions}

Figure 1: Schematic diagram showing a semiconducting sample of length $S$ enclosed between two plane-parallel ferromagnetic contacts. Illustrated are expression (3) for the thermoballistic current $J(x)$ and its analogue for the thermoballistic spin-polarized current $J_{-}(x)$.

Figure 2: The thermoballistic density $n(x)$ inside a homogeneous semiconductor for constant electric field, plotted versus $x / S$ (assuming $x_{1}=0$ ) for $\epsilon S=1$ and the indicated values of $l / S$. The density is normalized to the constant value it assumes in the diffusive limit $l / S \rightarrow 0$.

Figure 3: The zero bias $(\epsilon \rightarrow 0)$ current spin polarization $P_{J}(x)$ (assuming $\left.x_{1}=0\right)$ along a symmetric ferromagnet-semiconductor-ferromagnet structure with $S=1 \mu \mathrm{m}$ for the indicated values of the momentum relaxation length $l$. The solid curves correspond to zero interface resistance, the dashed curves to interface resistances of $10^{-7} \Omega \mathrm{cm}^{2}$ for spin-up electrons and $2 \times 10^{-7} \Omega \mathrm{cm}^{2}$ for spin-down electrons, respectively. For the remaining parameter values, see text.

Figure 4: The injected current spin polarization $P_{J}\left(x_{1}\right)$ for $S / L \rightarrow \infty$ as a function of the electric-field parameter $\epsilon$ for the indicated values of the momentum relaxation length $l$. The solid curves correspond to zero interface resistance, the dashed curves to interface resistances of $10^{-7} \Omega \mathrm{cm}^{2}$ for spin-up electrons and $2 \times 10^{-7} \Omega \mathrm{cm}^{2}$ for spin-down electrons, respectively. For the remaining parameter values, see text. 


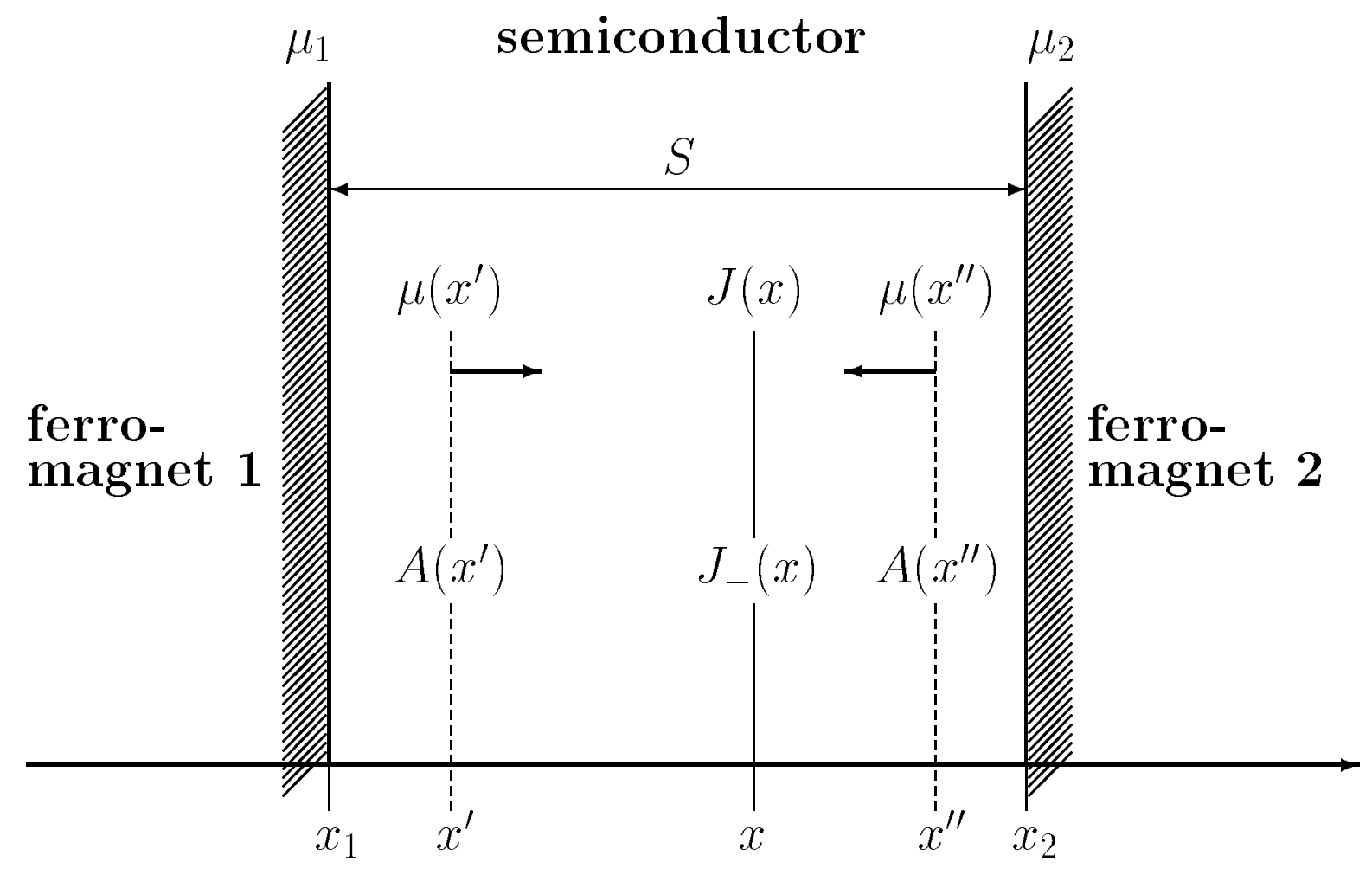

FIGURE 1 


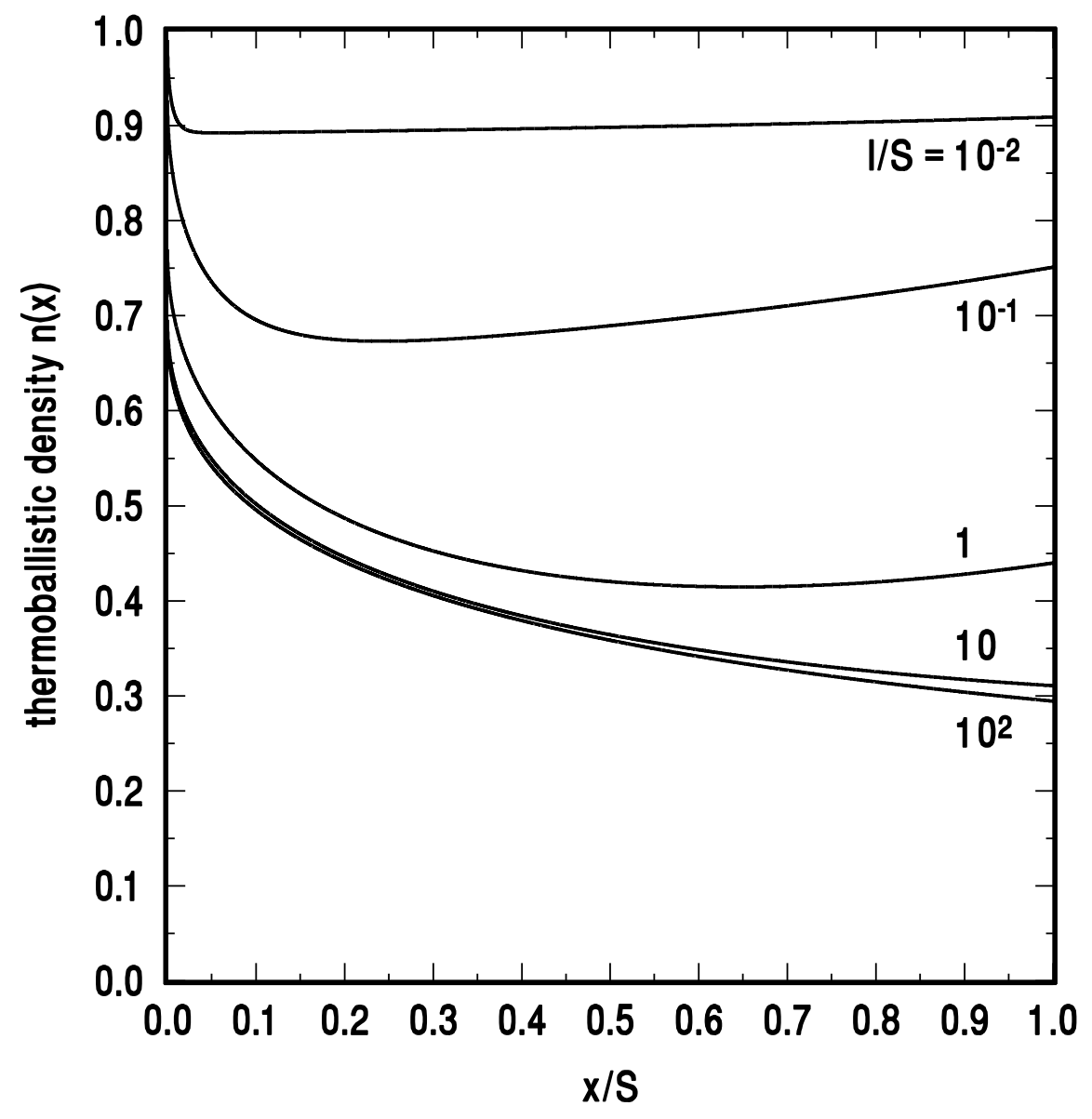

FIGURE 2 


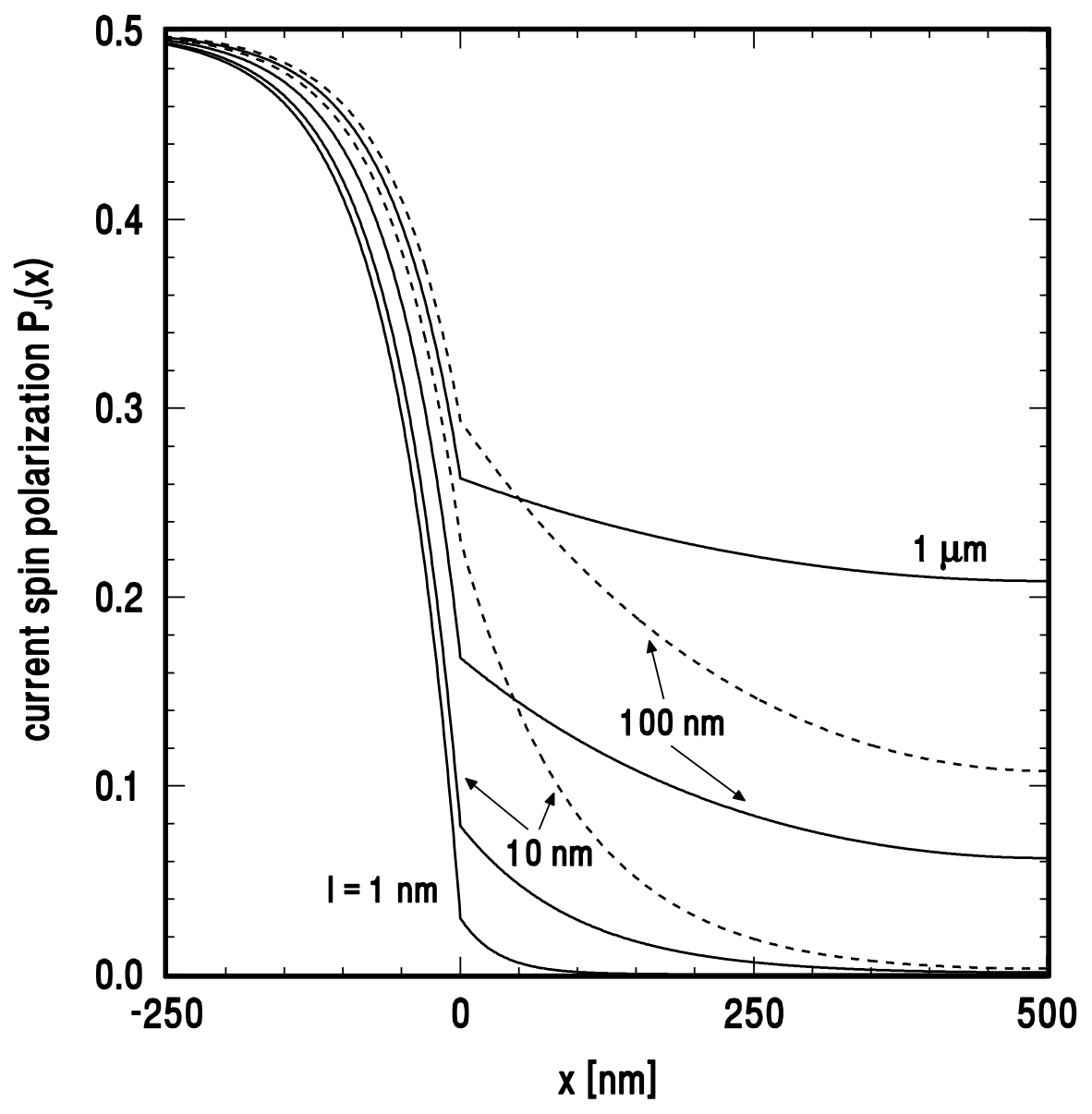

FIGURE 3 


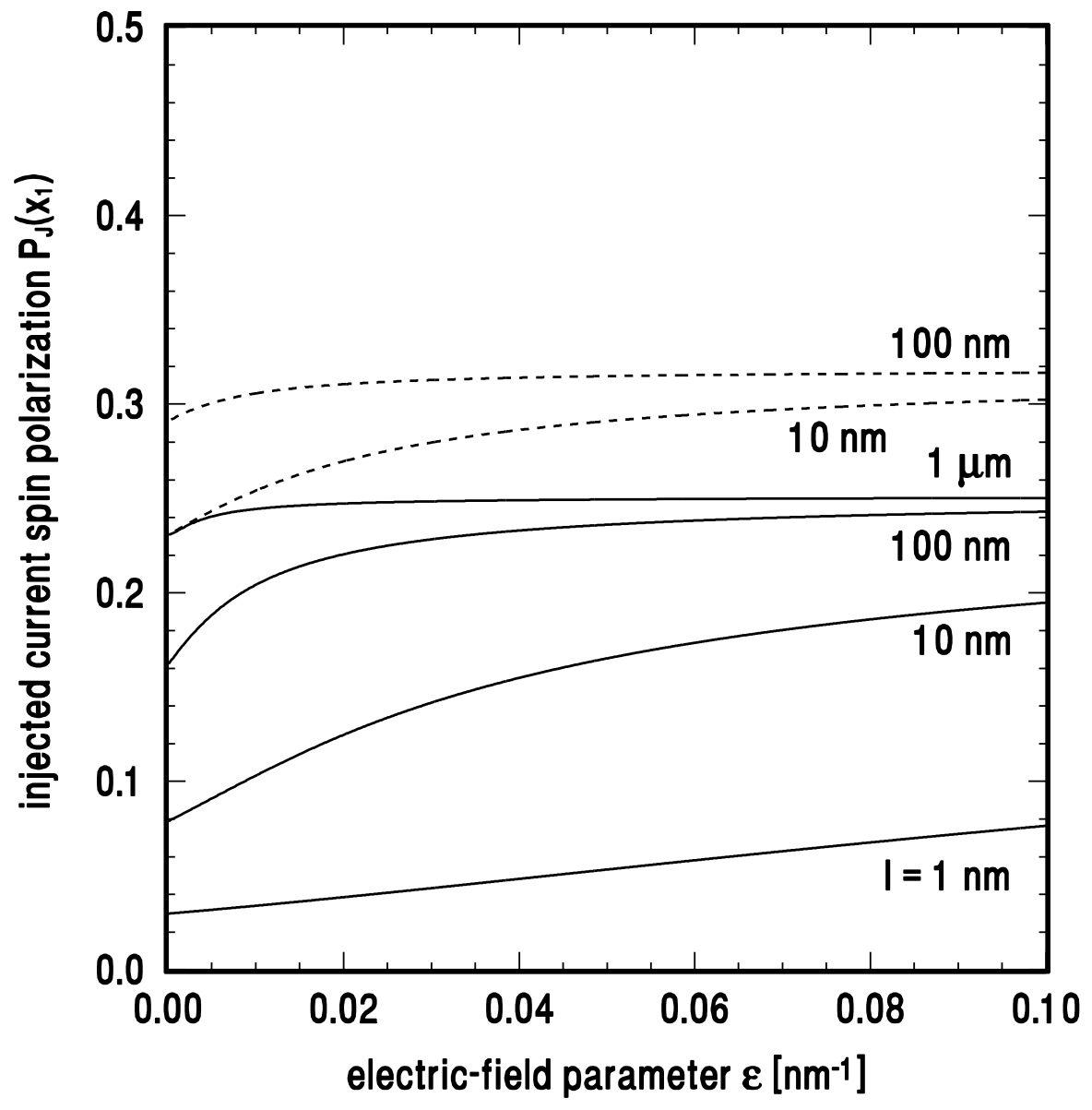

FIGURE 4 